\title{
UV-B辐射增强和氮沉降对入侵植物乌相生长的影响
}

\author{
邓邦良 刘 倩 刘喜帅 郑利亚 江亮波 郭晓敏 刘苑秋 张 令*
}

江西省森林培育重点实验室/江西特色林木资源培育与利用2011协同创新中心/江西农业大学林学院, 南昌 330045

摘 要 外来植物入侵是全球性问题, 严重威胁入侵地生态系统功能和稳定性。入侵植物与其在原产地生长特征的不同, 使 其在抵抗胁迫生境和利用资源方面具有较强的竞争能力, 从而具有较高的入侵潜力。UV-B辐射增强和氮沉降加剧作为两种 全球变化因子, 可能与土壤微生物共同作用于植物入侵的整个过程。了解UV-B辐射增强和氮沉降加剧是如何直接或间接影 响微生物介导的植物生长, 有助于揭示全球变化背景下的植物入侵机理, 为有效控制植物入侵并降低其对生态系统功能的危 害提供理论依据。该试验采用四因素裂区设计, 以入侵美国的中国乌柏(Triadica sebifera)为研究对象, 通过模拟UV-B辐射增 强和氮沉降加剧, 在土壤微生物控制条件下, 研究三者对不同种群乌柏生长的影响。结果显示: UV-B辐射增强、氮沉降加剧 和土壤微生物可能共同作用于乌柏成功入侵的整个过程。UV-B辐射增强导致乌柏形态学和生物量分配发生变化, 大部分的 资源分配给叶片以抵抗外界UV-B辐射胁迫。氮沉降使得乌柏将更多资源分配至地上部分, 特别是叶片, 减少了对地下生物量 的分配。原产地土壤微生物对乌柏生长具有显著正效应, 同时, 氮沉降增强了该效应而UV-B辐射增强对该效应没有影响。氮 沉降没有减缓UV-B辐射对乌柏的胁迫作用。入侵地乌柏种群相比于原产地乌柏种群在株高、叶生物量和总生物量方面已经 进化出了较为明显的优势, 此外, 入侵地乌柏种群相比于原产地乌柏种群减弱了在根冠比和根生物量方面对原产地土壤微生 物的依赖性, 但是增强了在叶面积比方面的依赖性。

关键词 非生物胁迫; 土壤微生物; 资源可利用性; 植物入侵

引用格式: 邓邦良, 刘倩, 刘喜帅, 郑利亚, 江亮波, 郭晓敏, 刘苑秋, 张令 (2017). UV-B辐射增强和氮沉降对入侵植物乌柏生长的影响. 植物生态学 报, 41, 471-479. doi: 10.17521/cjpe.2016.0292

\section{Effects of enhanced UV-B radiation and nitrogen deposition on the growth of invasive plant Triadica sebifera}

DENG Bang-Liang, LIU Qian, LIU Xi-Shuai, ZHENG Li-Ya, JIANG Liang-Bo, GUO Xiao-Min, LIU Yuan-Qiu, and ZHANG Ling*

Jiangxi Key Laboratory of Silviculture/Collaborative Innovation Center of Jiangxi Typical Trees Cultivation and Utilization, College of Forestry, Jiangxi Agricultural University, Nanchang 330045, China

\section{Abstract}

Aims Exotic plant invasions are important components of global change, threatening both the stability and function of invaded ecosystems. Shifts in competitive ability of invasive plants versus their native congeners have been documented. Enhanced UV-B radiation and nitrogen $(\mathrm{N})$ deposition might interact with soil biota communities impacting the invasion process of exotic plant species. To understand the potential effects by UV-B and $\mathrm{N}$ with soil biota on plant growth would enhance our understanding of the mechanisms in plant invasions in the context of global change.

Methods We conducted a full-factorial pot experiment in the native range (China) of Triadica sebifera invading US to investigate how UV-B radiation, $\mathrm{N}$ and soil biota together determined their seedling growth.

Important findings The results showed that UV-B radiation, $\mathrm{N}$ and soil sterilization together impacted the growth of $T$. sebifera seedlings. UV-B radiation induced changes in biomass allocation with larger leaf biomass observed in response to UV-B radiation. In addition, $\mathrm{N}$ increased aboveground biomass and decreased root biomass simultaneously. Soil biota imposed positive effects on growth of $T$. sebifera, and the addition of $\mathrm{N}$ amplified these positive effects. The negative effects by UV-B radiation on growth of $T$. sebifera showed no response to $\mathrm{N}$ addition. Plant height, leaf biomass and total biomass of the invasive T. sebifera populations out- performed those of the native ones. In addition, invasive $T$. sebifera populations weakened the dependence of root/shoot ratio and root biomass on local soil microorganisms than native populations, but enhanced that of leaf area ratio.

收稿日期Received: 2016-09-20 接受日期Accepted: 2017-01-03

* 通信作者Author for correspondence (E-mail: lingzhang09@126.com) 
Key words abiotic stress; soil microorganism; resource availability; plant invasion

Citation: Deng BL, Liu Q, Liu XS, Zheng LY, Jiang LB, Guo XM, Liu YQ, Zhang L (2017). Effects of enhanced UV-B radiation and nitrogen deposition on the growth of invasive plant Triadica sebifera. Chinese Journal of Plant Ecology, 41, 471-479. doi: 10.17521/cjpe.2016.0292

外来植物入侵是全球性问题, 不仅严重威胁入 侵地生态系统物种多样性, 还潜在影响生态系统过 程(如物质循环、群落演替)(类延宝等, 2010; 万方浩 等, 2011; 鞠瑞亭等, 2012; 陈宝明等, 2016)。当外来 植物被引入新的环境时, 由于天敌等外界因素的改 变, 一系列非生物和生物因素的交互作用使得选择 压力改变, 导致其中一部分外来植物对环境的响应 策略发生了改变并呈现出进化变异且具有较强的入 侵潜力, 这些入侵植物与其在原产地生长特征的不 同可能与非生物和生物因素息息相关(Blossey \& Notzold, 1995; Henery et al., 2010; 刘建等, 2010)。

由于氟氯烃等大气污染物的大量排放, 致使 $\mathrm{O}_{3}$ 层空洞日趋严重, 最终导致地表UV-B辐射强度增 加(Williamson et al., 2014)。UV-B辐射增强会造成植 物DNA损伤(如嘧啶二聚体)并诱发一系列形态学和 生理生化方面的防御反应, 甚至通过干扰植物-土 壤反馈过程而进一步影响土壤养分吸收和微生物活 性等过程(陈慧泽和韩榕, 2015; 李良博等, 2015; Suchar \& Robberecht, 2016)。由于适应策略以及响应 机制的不同, 使得入侵植物与本地植物对UV-B辐 射损伤的修复和抵御能力不同(Hock et al., 2015; 李 良博等, 2015, 2016; Wang et al., 2015), 因此, UV-B作 为一种非生物胁迫, 可能在植物入侵过程中扮演着 至关重要的角色。

氮作为植物生长所需的大量元素, 其水平的高 低直接影响植物的新陈代谢和生长发育。工业革命 以来, 人类活动导致大气氮沉降加剧(Kanakidou et al., 2016), 直接提高了土壤氮素可利用水平。“资 源可利用性增强”假说认为: 某一群落内资源可利 用水平增加会导致外来植物入侵该群落的可能性增 加(Davis et al., 2000)。由于部分入侵植物较之本地 植物表现出更强的氮吸收速率(Zou et al., 2006), 因 而氮沉降加剧可能对植物入侵具有促进作用 (Bradley et al., 2010; 陈彤等, 2012)。

“天敌逃逸”假说认为, 外来植物能够成功入侵 可能与入侵地无专食性天敌危害有关(Keane \& Crawley, 2002; Huang et al., 2012; 黄伟等, 2013), 随着研究的深入, 土壤有害微生物(如病原菌)也被 认为具有类似于地上天敌的作用(van der Putten et al., 2007; Callaway et al., 2011); 此外, 土壤有益微 生物共生体(如根瘤菌、菌根真菌等)通常能够协助 入侵植物高效地利用土壤氮(Huang et al., 2016)、磷 (Zhang et al., 2013)等养分, 因此, 土壤有害和有益 微生物的综合效应可能促进或者阻碍植物入侵。

乌柏(Triadica sebifera) 原产于中国, 18世纪末 作为油料和观赏树种引入美国, 如今在美国东南部 出现严重入侵现象, 形成茂密的单一优势种, 严重 破坏入侵地生态系统(Huang et al., 2012; Zhang et al., 2013; Yang et al., 2015)。乌柏入侵种群通过“地上优 先”的分配策略以及高耐性、低抗性的进化选择，使 其比原产地种群具有更高的相对生长速率、氮吸收 速率和比叶面积等特征(Zou et al., 2006, 2007; Zhang et al., 2013; 吴昊和丁建清, 2014; Yang et al., 2015), 在生长方面已经进化出明显的优势。鉴于乌柏成功 入侵与非生物和生物因素的复杂性, 国内外学者相 继开展了原产地和入侵地乌柏种群对UV-B辐射增强 (Wang et al., 2015), 氮沉降和土壤微生物交互作用 (Zhang et al., 2013), 施肥、盐度和土壤微生物交互作 用(Yang et al., 2015)等相关研究, 通过了解不同非生 物和生物条件下入侵地和原产地乌柏种群在形态学 和生理特征方面的差异, 逐步揭示了乌柏成功入侵 的关键因素, 然而, 土壤肥力是如何直接或间接调控 微生物介导的植物生长来应对非生物胁迫尚不明确。

因此, 本研究以盆栽试验方式, 通过观察原产 地(中国)和入侵地(美国)乌柏种群在UV-B辐射增 强、氮沉降加剧和土壤灭菌三者共同作用下的形态 学与生理特征差异, 用于了解UV-B辐射和氮沉降 加剧是如何直接或间接影响微生物介导的乌柏生 长。拟解决问题如下: 1)氮沉降和UV-B辐射是否与 土壤灭菌相互作用于乌柏生长？2)氮沉降是否会增 加乌柏对UV-B辐射的耐受力？3)不同处理组合对 原产地和入侵地乌柏种群的生长影响有何差异?

\section{1 材料和方法}

\section{1 盆栽土壤}

采集乌柏非根际土壤并移除石块和其他杂物, 
过篮处理, 备用。对土壤进行基本背景值分析, 有机 质碳含量为 $(4.89 \pm 0.15) \mathrm{g} \cdot \mathrm{kg}^{-1}$, 全氮含量为 $(0.47 \pm$ $0.01) \mathrm{g} \cdot \mathrm{kg}^{-1}$, 铵态氮 $\left(\mathrm{NH}_{4}^{+}-\mathrm{N}\right)$ 含量为 $(10.32 \pm$ $0.10) \mathrm{mg} \cdot \mathrm{kg}^{-1}$, 硝态氮 $\left(\mathrm{NO}_{3}^{-}-\mathrm{N}\right)$ 含量为 $(3.39 \pm$ $0.06) \mathrm{mg} \cdot \mathrm{kg}^{-1}$, 有效磷含量为 $(2.6 \pm 0.65) \mathrm{mg} \cdot \mathrm{kg}^{-1}$, $\mathrm{pH}$ 值为 $5.39 \pm 0.02$ 。土壤灭菌采用高压蒸汽灭菌法 $\left(121{ }^{\circ} \mathrm{C}, 45 \mathrm{~min}\right)$ 。

\section{2 乌相种子采集和育苗}

2014年, 在中国(原产地)和美国(入侵地)分别 采集 6 个种群的乌柏种子, 共 12 个种群(表1), 每个 种群的种子至少采集于3株树上。2015年4月份按照 种源分类, 分别均匀地种植于灭菌的非根际乌柏土 壤里进行育苗, 期间通过浇酒蒸馏水使土壤保持湿 润。待6月初乌柏幼苗萌发至适合移栽时(35天左右), 选择株高相近的乌柏幼苗, 然后按照土壤灭菌和非 灭菌处理, 将乌柏幼苗分别移栽于对应的装有灭菌 和非灭菌土壤的花盆(体积: $1.2 \mathrm{~L}$, 填装土壤质量: 1 $\mathrm{kg})$ 中。

\section{3 试验设计}

试验为四因素裂区设计, 其中UV-B辐射增强 (对照vs.UV-B辐射增强20\%)为主处理, 氮沉降(对 照 $v s$.氮沉降) $\times$ 土壤灭菌 (对照 $v s$. 土壤灭菌) $\times$ 种源 (原产地vs. 入侵地, 各包含 6 个种群, 种群为种源内 部嵌套因子)为副处理, 重复 4 次, 共 $2 \times 2 \times 2 \times$ $(6+6) \times 4=384$ 盆乌柏。通过安装紫外线灯管进行 UV-B辐射增补(倪郁等, 2015)。用 $\mathrm{NH}_{4} \mathrm{NO}_{3}$ 溶液模拟 $8 \mathrm{~g} \mathrm{~N} \cdot \mathrm{m}^{-2}$ 的氮沉降, 分3次施入, 每次加液 $5 \mathrm{~mL}$ (相 当于 $0.03 \mathrm{~g} \mathrm{~N} \cdot \mathrm{kg}^{-1}$ 土壤)。

表1 不同乌柏种源的地理分布信息

Table 1 Locations of native and invasive Triadica sebifera populations used in the study

\begin{tabular}{llcc}
\hline & 种群 Population & 经度 Longitude & 纬度 Latitude \\
\hline 中国 China & 福建 Fujian & $119.28^{\circ} \mathrm{E}$ & $26.07^{\circ} \mathrm{N}$ \\
& 广东 Guangdong & $112.38^{\circ} \mathrm{E}$ & $24.78^{\circ} \mathrm{N}$ \\
& 贵州 Guizhou & $106.88^{\circ} \mathrm{E}$ & $27.08^{\circ} \mathrm{N}$ \\
& 湖北 Hubei & $110.73^{\circ} \mathrm{E}$ & $32.05^{\circ} \mathrm{N}$ \\
& 江苏 Jiangsu & $118.37^{\circ} \mathrm{E}$ & $31.23^{\circ} \mathrm{N}$ \\
& 江西 Jiangxi & $117.12^{\circ} \mathrm{E}$ & $28.45^{\circ} \mathrm{N}$ \\
& Alabama & $88.15^{\circ} \mathrm{W}$ & $30.58^{\circ} \mathrm{N}$ \\
& Georgia & $81.01^{\circ} \mathrm{W}$ & $32.01^{\circ} \mathrm{N}$ \\
& Louisiana 1 & $93.15^{\circ} \mathrm{W}$ & $30.23^{\circ} \mathrm{N}$ \\
& Louisiana 2 & $92.02^{\circ} \mathrm{W}$ & $30.25^{\circ} \mathrm{N}$ \\
& Texas 1 & $95.03^{\circ} \mathrm{W}$ & $29.78^{\circ} \mathrm{N}$ \\
& Texas 2 & $95.45^{\circ} \mathrm{W}$ & $28.97^{\circ} \mathrm{N}$ \\
\hline
\end{tabular}

\section{4 试验方法}

有机质碳测定采用重铬酸钾容量法(鲁如坤, 2000); 全氮测定采用半微量凯氏消化法 $\left(\mathrm{Kjeltec}^{\mathrm{TM}}\right.$ 8400 Analyzer Unit, FOSS, Hillerød, Denmark); $\mathrm{NH}_{4}^{+}-\mathrm{N} 、 \mathrm{NO}_{3}^{-}-\mathrm{N}$ 和有效磷分别采用靛酚蓝比色法、 双波长紫外比色法 $\left(\mathrm{A}_{220}-\mathrm{A}_{275}\right)$ 和钼锑抗比色法(鲁如 坤, 2000)(UV-6300型紫外可见分光光度计, MAPADA, 上海) 测定; $\mathrm{pH}$ 值采用电极法 (土: 水质量比为 1:2.5)(LE438 pH, METTER TOLEDO，上海)。

2015年10月底, 测定植株株高(收获前), 随后 收获植株并测定总叶片数、总叶面积、叶片生物量、 茎生物量、根生物量、地上生物量(叶生物量+茎生 物量)和总生物量等指标, 通过以上指标进行比叶 面积 $\left(S L A\right.$, 植物单位叶干质量叶面积, $\left.\mathrm{cm}^{2} \cdot \mathrm{g}^{-1}\right)$, 叶 面积比 $\left(L A R\right.$, 植物单位总干质量叶面积, $\left.\mathrm{cm}^{2} \cdot \mathrm{g}^{-1}\right)$ 和 根冠比( $R S R$, 地下生物量: 地上生物量)等的换算。总 叶面积通过扫描新鲜叶片, 然后使用 SCNIMAGE (Scion Corporation, www.scioncorp.com)(Zhang et al., 2013)软件测得。生物量通过 $60{ }^{\circ} \mathrm{C}$ 下烘干至恒质量 称量测定。

\section{5 数据处理}

统计分析使用JMP 9.0软件, 多重比较采用最 小显著差异法 $(L S D, \alpha=0.05)$ 。绘图使用Origin 8.1 软件。

\section{2 结果}

\section{1 形态学指标分析}

土壤灭菌、氮沉降以及土壤灭菌和氮沉降交互 作用对株高具有极显著影响 $(p<0.01)$ (表2)。氮沉降 对株高具有显著正效应, 土壤灭菌以及土壤灭菌和 氮沉降交互作用对株高具有显著负效应; 在氮沉降 条件下, 土壤灭菌对株高具有显著负效应; 在土壤灭 菌条件下, 氮沉降对株高没有影响(图1A)。入侵地乌 柏较之原产地乌柏具有较高的株高(+13.17\%)(图1B)。

土壤灭菌以及土壤灭菌和氮沉降交互作用对 叶片数具有极显著影响 $(p<0.01)$, UV-B辐射对叶片 数具有显著影响 $(p<0.05)$ (表2)。氮沉降对叶片数具 有显著正效应, 土壤灭菌以及土壤灭菌和氮沉降的 交互作用对叶片数具有显著负效应; 在氮沉降条件 下, 土壤灭菌对叶片数具有显著负效应; 在土壤灭 菌条件下, 氮沉降对叶片数没有影响(图2A)。UV-B 辐射增加叶片数( $+5.59 \%)$ (图2B)。 
表2 UV-B辐射 $(U V)$ 、土壤灭菌 $(\mathrm{S})$ 和氮沉降 $(\mathrm{N})$ 处理对不同种源 $(\mathrm{O})$ 乌柏形态学特征的影响方差分析

Table 2 The dependence of morphological traits on Triadica sebifera origin (O), UV-B radiation (UV), soil sterilization (S) and nitrogen deposition (N) treatment, and their interactions in ANOVAs

\begin{tabular}{|c|c|c|c|c|c|c|}
\hline \multirow[t]{2}{*}{ 处理 Treatment } & \multirow[t]{2}{*}{$d f$} & \multicolumn{5}{|c|}{$F$ value } \\
\hline & & 株高 Plant height & 叶片数 Leaf number & 叶面积 Leaf areas & 比叶面积 Specific leaf area & 叶面积比 Leaf area ratio \\
\hline UV & 1 & 0.01 & $4.96^{*}$ & 0.07 & $5.19^{*}$ & 0.00 \\
\hline S & 1 & $144.85^{* *}$ & $343.02^{* *}$ & $820.08^{* *}$ & $14.66^{* *}$ & $78.98^{* *}$ \\
\hline $\mathrm{N}$ & 1 & $37.90^{* *}$ & 3.60 & $40.55^{* *}$ & 2.03 & $59.97^{* *}$ \\
\hline $\mathrm{O}$ & 1 & $58.13^{* *}$ & 0.76 & 1.20 & 0.16 & 1.82 \\
\hline $\mathrm{UV} \times \mathrm{S}$ & 1 & 0.52 & 0.26 & 0.02 & 0.05 & 0.58 \\
\hline $\mathrm{UV} \times \mathrm{N}$ & 1 & 2.46 & 2.58 & 2.27 & 0.00 & 1.21 \\
\hline $\mathrm{UV} \times \mathrm{O}$ & 1 & 0.05 & 0.22 & 0.20 & 1.01 & 0.05 \\
\hline $\mathrm{S} \times \mathrm{N}$ & 1 & $19.70^{* *}$ & $18.90^{* *}$ & $73.34^{* *}$ & 0.11 & $44.87^{* *}$ \\
\hline $\mathrm{S} \times \mathrm{O}$ & 1 & 0.22 & 1.86 & 2.09 & 2.59 & $8.57^{* *}$ \\
\hline $\mathrm{N} \times \mathrm{O}$ & 1 & 0.01 & 0.28 & 0.52 & 0.01 & 1.12 \\
\hline $\mathrm{UV} \times \mathrm{S} \times \mathrm{N}$ & 1 & 0.01 & 2.49 & 0.06 & 0.23 & 0.10 \\
\hline $\mathrm{UV} \times \mathrm{S} \times \mathrm{O}$ & 1 & 0.75 & 1.12 & 0.22 & 0.46 & 0.16 \\
\hline $\mathrm{UV} \times \mathrm{N} \times \mathrm{O}$ & 1 & 0.07 & 0.08 & 0.24 & 0.12 & 0.40 \\
\hline $\mathrm{S} \times \mathrm{N} \times \mathrm{O}$ & 1 & 1.17 & 2.18 & 2.76 & 1.52 & 2.81 \\
\hline $\mathrm{UV} \times \mathrm{S} \times \mathrm{N} \times \mathrm{O}$ & 1 & 0.37 & 0.01 & 1.05 & 0.12 & 0.46 \\
\hline
\end{tabular}

$*, 0.01<p<0.05, * *, p<0.01$, 显著性结果 $(p<0.05)$ 以粗体形式展示。

$*, 0.01<p<0.05,{ }^{* *}, p<0.01$, significant results $(p<0.05)$ are shown in bold.
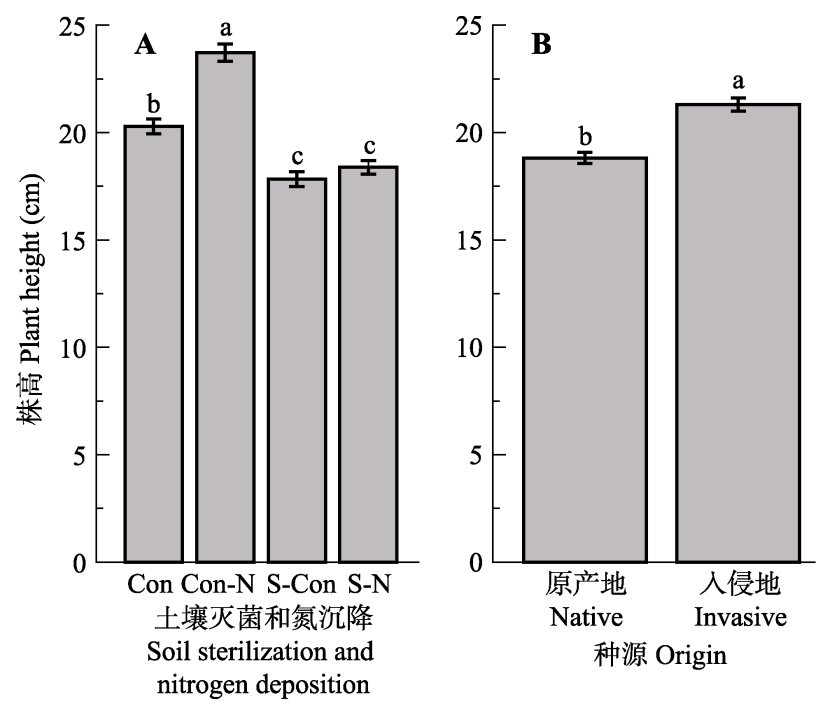

图1 不同处理对乌柏株高的影响(平均值土标准误差)。Con, 对照; $\mathrm{N}$, 氮沉降; S, 土壤灭菌。相同小写字母表示没有显著 差异 $(p>0.05, L S D$ test $)$ 。

Fig. 1 Effect of different treatments on plant height of Triadica sebifera (mean $\pm S E$ ). Con, control; N, nitrogen deposition; S, soil sterilization. Same lowercase letters indicate no significant differences ( $p>0.05, L S D$ test).

土壤灭菌、氮沉降以及两者交互作用对叶面积 具有极显著影响 $(p<0.01)$ (表2)。氮沉降对叶面积具 有显著正效应，土壤灭菌以及土壤灭菌和氮沉降的 交互作用对叶面积具有显著负效应; 在氮沉降条件 下，土壤灭菌对叶面积具有显著负效应; 在土壤灭
菌条件下，氮沉降对叶面积没有影响(图2C)。

UV-B辐射对 $S L A$ 具有显著影响 $(p<0.05)$, 土壤 灭菌对 $S L A$ 具有极显著影响 $(p<0.01)$ (表2)。UV-B辐 射显著降低SLA (-5.08\%)(图2D)。土壤灭菌显著降 低SLA (-9.41\%)(图2E)。

土壤灭菌、氮沉降以及两者交互作用对 $L A R$ 具 有极显著影响 $(p<0.01)$ (表2)。氮沉降对 $L A R$ 具有显 著正效应，土壤灭菌以及土壤灭菌和氮沉降交互作 用对 $L A R$ 没有影响; 氮沉降条件下，土壤灭菌对 $L A R$ 具有显著负效应; 在土壤灭菌条件下, 氮沉降 对LAR没有影响(图2F)。对照条件下, 原产地乌柏和 入侵地乌柏 $L A R$ 没有差异; 土壤灭菌条件下, 原产 地乌柏较之入侵地乌柏具有较高的 $L A R(+16.05 \%)$; 土壤灭菌对原产地和入侵地乌柏 $L A R$ 都具有显著负 效应，但是对原产地乌柏的负效应低于对入侵地乌 柏的负效应(-15.97\% vs. -30.53\%)(图2G)。

\section{2 生物量分析}

土壤灭菌、氮沉降以及两者交互作用对叶生物 量具有极显著影响 $(p<0.01), \mathrm{UV}-\mathrm{B}$ 辐射对叶生物量 具有显著影响 $(p<0.05)$ (表3)。氮沉降对叶生物量具 有显著正效应, 土壤灭菌以及土壤灭菌和氮沉降交 互作用对叶生物量具有显著负效应; 在氮沉降条件 下, 土壤灭菌对叶生物量具有显著负效应; 在土壤 


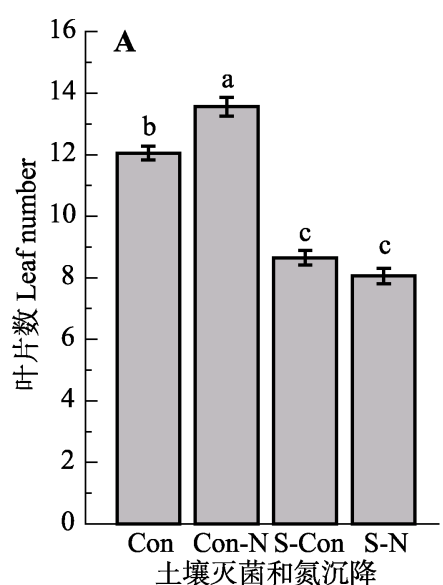

Soil sterilization and nitrogen deposition

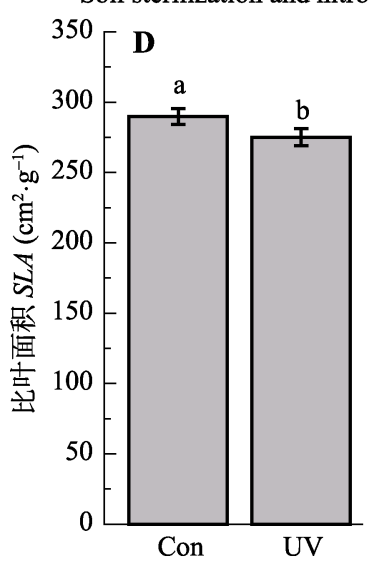

UV-B 辐射

UV-B radiation

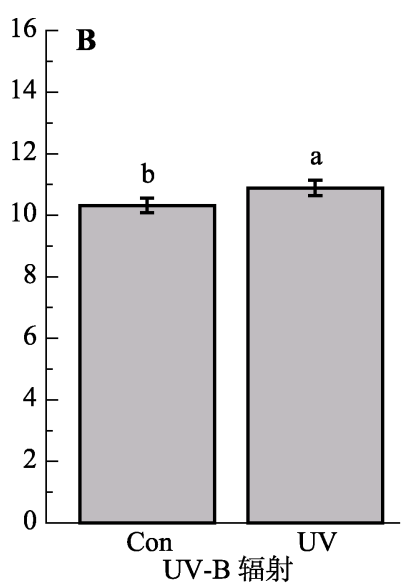

UV-B radiation

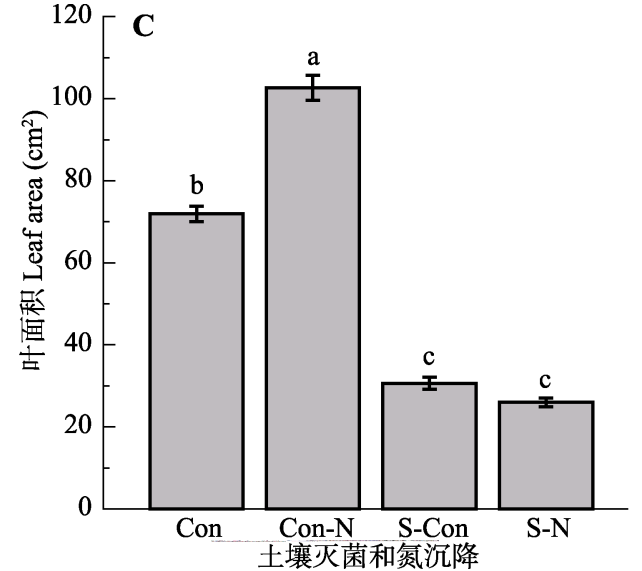

Soil sterilization and nitrogen deposition

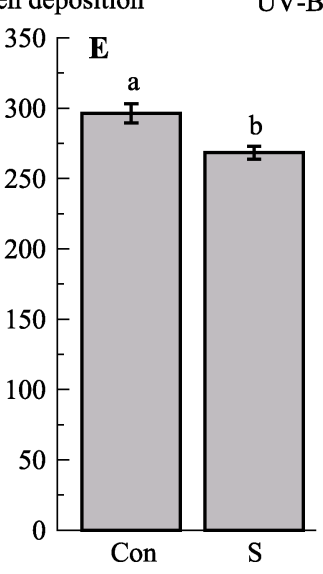

土壤灭菌

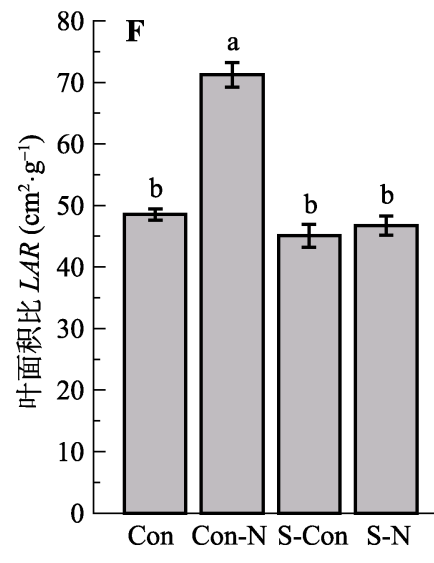

土壤灭菌和氮沉降

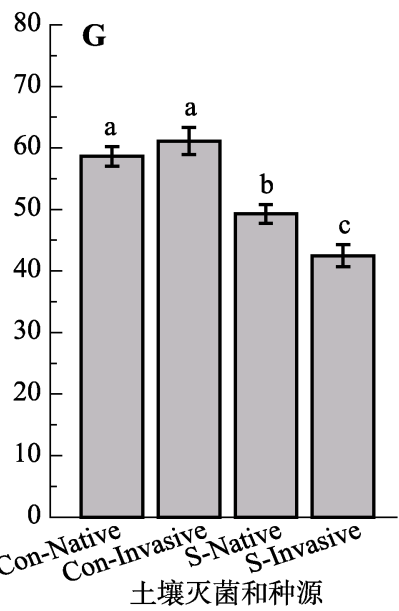

土壤灭菌和种源

Soil sterilization and nitrogen deposition Soil sterilization and origin

图2 不同处理对乌柏叶形态学特征的影响(平均值土标准误差)。Con, 对照; N, 氮沉降; S, 土壤灭菌; UV, UV-B辐射; Native, 原产地; Invasive, 入侵地; $S L A$, 比叶面积; $L A R$, 叶面积比。相同小写字母表示没有显著差异 $(p>0.05, L S D$ test)。

Fig. 2 Effect of different treatments on leaf morphological traits of Triadica sebifera (mean $\pm S E$ ). Con, control; N, nitrogen deposition; S, soil sterilization; UV, UV-B radiation; $S L A$, specific leaf area; $L A R$, leaf area ratio. Same lowercase letters indicate no significant differences ( $p>0.05, L S D$ test).

表3 UV-B辐射(UV)、土壤灭菌(S)和氮沉降 $(\mathrm{N})$ 处理对不同种源 $(\mathrm{O})$ 乌柏生物量的影响方差分析

Table 3 The dependence of biomass on Triadica sebifera origin (O), UV-B radiation (UV), soil sterilization (S) and nitrogen deposition (N) treatment, and their interactions in ANOVAs

\begin{tabular}{|c|c|c|c|c|c|c|c|}
\hline \multirow[t]{2}{*}{ 处理 Treatment } & \multirow[t]{2}{*}{$d f$} & \multicolumn{6}{|c|}{$F$ value } \\
\hline & & $\begin{array}{c}\text { 叶生物量 } \\
\text { Leaf biomass }\end{array}$ & $\begin{array}{c}\text { 茎生物量 } \\
\text { Stem biomass }\end{array}$ & $\begin{array}{c}\text { 根生物量 } \\
\text { Root biomass }\end{array}$ & $\begin{array}{c}\text { 地上生物量 } \\
\text { Aboveground biomass }\end{array}$ & $\begin{array}{c}\text { 总生物量 } \\
\text { Total biomass }\end{array}$ & $\begin{array}{c}\text { 根冠比 } \\
\text { Root/shoot ratio }\end{array}$ \\
\hline UV & 1 & $4.12^{*}$ & 0.73 & 1.32 & 0.10 & 0.94 & 0.37 \\
\hline S & 1 & $966.67^{* *}$ & $794.51^{* *}$ & $690.14^{* *}$ & $1007.44^{* *}$ & $1059.72^{* *}$ & $4.17^{*}$ \\
\hline $\mathrm{N}$ & 1 & $33.38^{* *}$ & 0.26 & $53.60^{* * *}$ & $12.80^{* *}$ & $10.08^{* *}$ & $109.60^{* *}$ \\
\hline $\mathrm{O}$ & 1 & 0.00 & $6.76^{* *}$ & 3.73 & 3.80 & $4.33^{*}$ & 2.64 \\
\hline $\mathrm{UV} \times \mathrm{S}$ & 1 & 0.01 & 0.00 & 0.98 & 0.01 & 0.35 & 2.51 \\
\hline $\mathrm{UV} \times \mathrm{N}$ & 1 & 2.49 & 2.37 & 0.12 & 3.35 & 1.21 & 1.79 \\
\hline $\mathrm{UV} \times \mathrm{O}$ & 1 & 1.16 & 0.06 & 0.04 & 0.44 & 0.06 & 0.91 \\
\hline $\mathrm{S} \times \mathrm{N}$ & 1 & $62.76^{* *}$ & $18.38^{* *}$ & 2.37 & $47.51^{* *}$ & $5.96^{*}$ & $46.14^{* *}$ \\
\hline $\mathrm{S} \times \mathrm{O}$ & 1 & 0.20 & 0.00 & $7.46^{* *}$ & 0.42 & 2.24 & $14.58^{* *}$ \\
\hline $\mathrm{N} \times \mathrm{O}$ & 1 & 0.02 & 1.57 & 1.54 & 0.25 & 1.30 & 0.06 \\
\hline $\mathrm{UV} \times \mathrm{S} \times \mathrm{N}$ & 1 & 0.07 & 0.04 & 0.00 & 0.19 & 0.00 & 0.07 \\
\hline $\mathrm{UV} \times \mathrm{S} \times \mathrm{O}$ & 1 & 0.02 & 0.04 & 0.00 & 0.01 & 0.00 & 0.85 \\
\hline $\mathrm{UV} \times \mathrm{N} \times \mathrm{O}$ & 1 & 0.20 & 0.23 & 0.32 & 0.67 & 0.37 & 0.00 \\
\hline $\mathrm{S} \times \mathrm{N} \times \mathrm{O}$ & 1 & 3.50 & 0.27 & 0.08 & 1.31 & 0.75 & 0.99 \\
\hline $\mathrm{UV} \times \mathrm{S} \times \mathrm{N} \times \mathrm{O}$ & 1 & 1.49 & 1.12 & 0.01 & 1.52 & 0.37 & 1.55 \\
\hline
\end{tabular}

$*, 0.01<p<0.05 ; * *, p<0.01$; 显著性结果 $(p<0.05)$ 以粗体形式展示。

*, $0.01<p<0.05 ; * *, p<0.01$; significant results $(p<0.05)$ are shown in bold. 
灭菌条件下, 氮沉降对叶生物量没有影响(图3A)。 UV-B辐射增加叶生物量(+5.53\%)(图3B)。

土壤灭菌、氮沉降以及两者交互作用对茎生物 量具有极显著影响 $(p<0.01)$ (表3)。氮沉降对茎生物 量具有显著正效应, 土壤灭菌以及土壤灭菌和氮沉 降交互作用对茎生物量具有显著负效应; 在氮沉降 条件下, 土壤灭菌对茎生物量具有显著负效应; 在 土壤灭菌条件下, 氮沉降对茎生物量具有显著负效 应(图3C)。入侵地乌柏较之原产地乌柏具有较高的 茎生物量 $(+7.35 \%)$ (图3D)。

土壤灭菌以及氮沉降都对根生物量具有极显 著影响 $(p<0.01)$ (表3)。对照条件下, 原产地乌柏和 入侵地乌柏根生物量没有差异; 土壤灭菌条件下,
入侵地乌柏较之原产地乌柏具有较高的根生物量 (+23.82\%); 土壤灭菌对原产地和入侵地乌柏根生 物量都具有显著负效应, 但是对原产地乌柏的负效 应高于对入侵地乌柏的负效应(-63.27\% vs. $-53.64 \%)$ (图3E)。氮沉降降低根生物量 (-20.68\%)(图3F)。

土壤灭菌、氮沉降以及两者交互作用对地上生 物量具有极显著影响 $(p<0.01)$ (表3)。氮沉降对地上 生物量具有显著正效应, 土壤灭菌以及土壤灭菌和 氮沉降交互作用对地上生物量具有显著负效应; 在 氮沉降条件下, 土壤灭菌对地上生物量具有显著负 效应; 在土壤灭菌条件下, 氮沉降对地上生物量没 有影响(图3G)。

入侵地乌柏较之原产地乌柏具有较高的总生
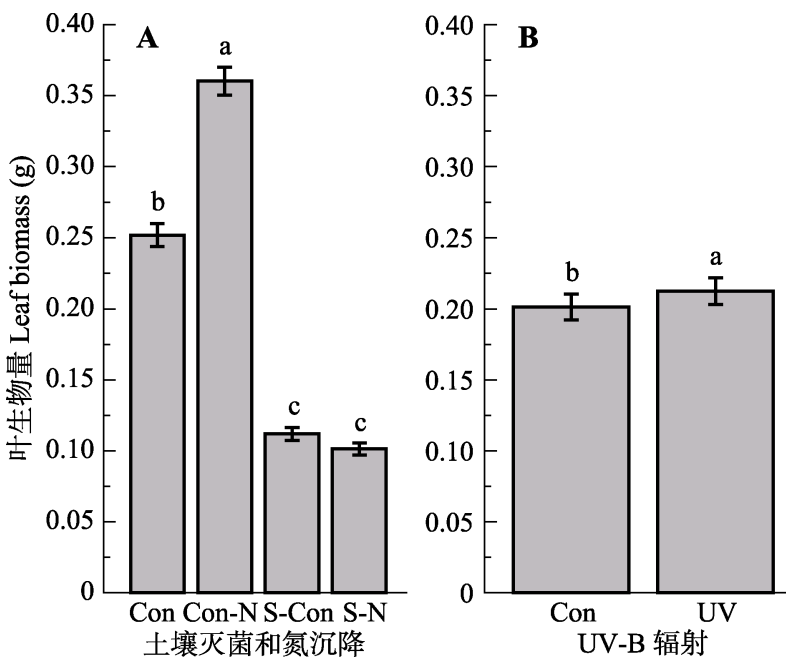

Soil sterilization and nitrogen deposition

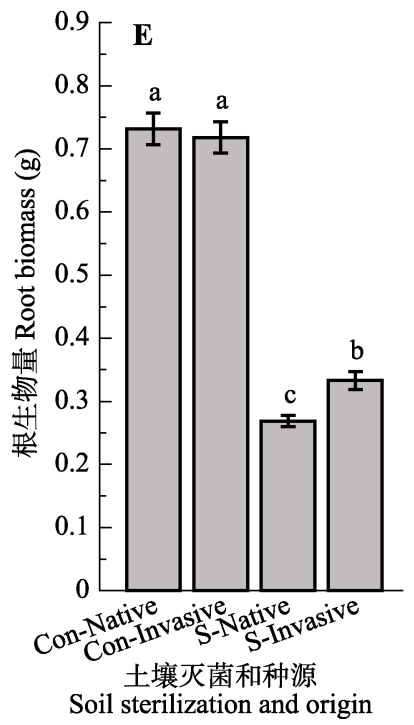

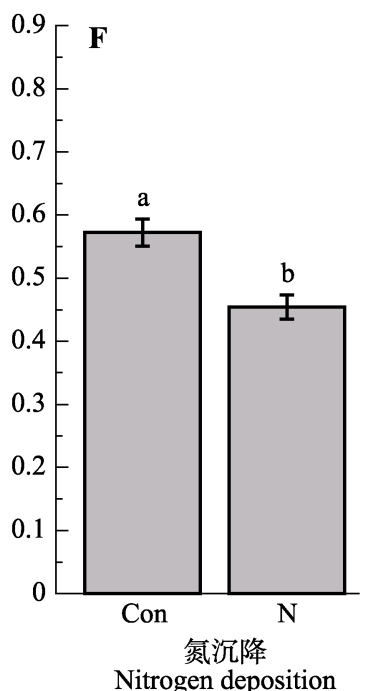

Nitrogen deposition
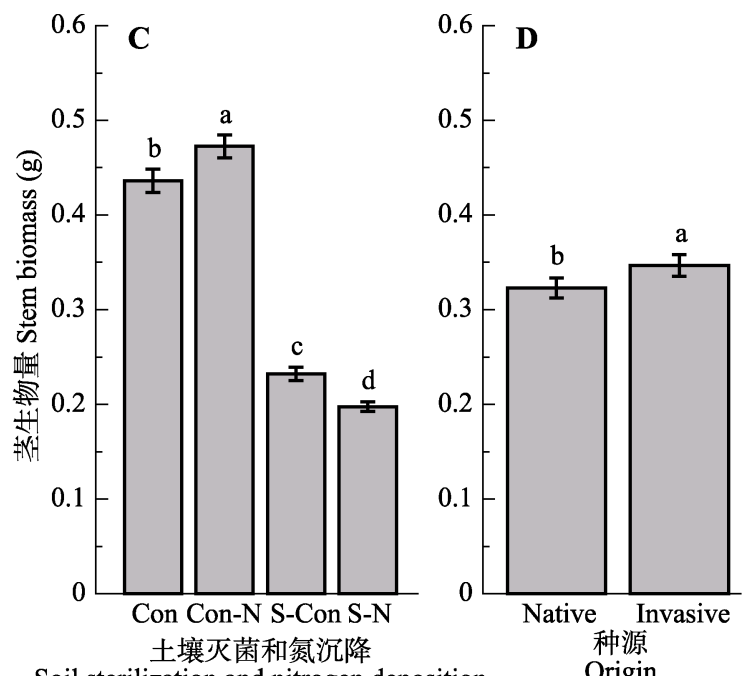

Soil sterilization and nitrogen deposition
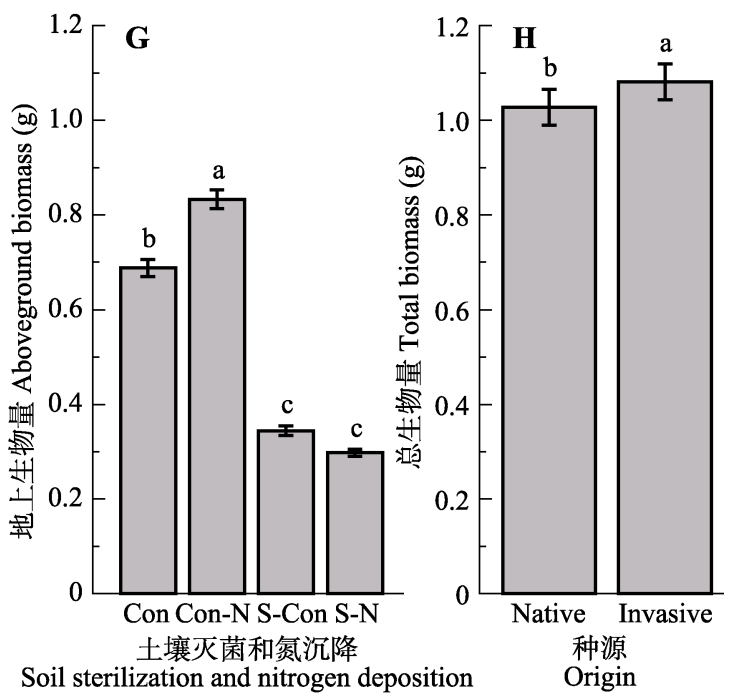

图3 不同处理对乌柏生物量的影响(平均值土标准误差)。Con, 对照; N, 氮沉降; S, 土壤灭菌; UV, UV-B辐射; Native, 原产地; Invasive, 入侵地。相同小写字母表示没有显著差异 ( $p>0.05, L S D$ test)。

Fig. 3 Effect of different treatments on biomass of Triadica sebifera (mean $\pm S E$ ). Con, control; N, nitrogen deposition; S, soil sterilization; UV, UV-B radiation. Same lowercase letters indicate no significant differences ( $p>0.05, L S D$ test).

www.plant-ecology.com 
物量 $(+5.29 \%)$ (图3H)。

氮沉降以及土壤灭菌和氮沉降交互作用都对 $R S R$ 具有极显著影响 $(p<0.01)$, 土壤灭菌对 $R S R$ 具 有显著影响 $(p<0.05)$ (表3)。氮沉降、土壤灭菌以及 两者的交互作用对 $R S R$ 具有显著负效应; 在氮沉降 条件下, 土壤灭菌增加 $R S R$; 在土壤灭菌条件下, 氮 沉降降低RSR (图4A)。对照条件下, 原产地乌柏和 入侵地乌柏 $R S R$ 没有差异; 土壤灭菌条件下, 入侵 地乌柏较之原产地乌柏具有较高的 $R S R(+15.81 \%)$; 土壤灭菌只对原产地乌柏 $R S R$ 具有显著负效应, 对 入侵地乌柏 $R S R$ 没有影响(图4B)。

\section{3 讨论}

\section{1 氮沉降增强乌柏与土壤微生物间的正效应}

株高、叶片数、叶面积、 $L A R$ 、叶生物量、茎 生物量、地上生物量、总生物量和 $R S R$ 都受到氮沉 降和土壤灭菌的交互作用影响(表2, 表3)。对照处理 比灭菌处理更容易受到氮沉降对乌柏株高(图1A)、 叶片数(图2A)、LAR (图2F)、叶生物量(图3A)、茎 生物量(图3C)和地上生物量(图3G)特征的增强影 响。换言之, 氮沉降增强了乌柏与土壤微生物间的 正效应。此外, 氮沉降显著增加了乌柏株高(图1A)、 叶片数(图2A)、叶面积(图2C)、LAR (图2F)、叶生物
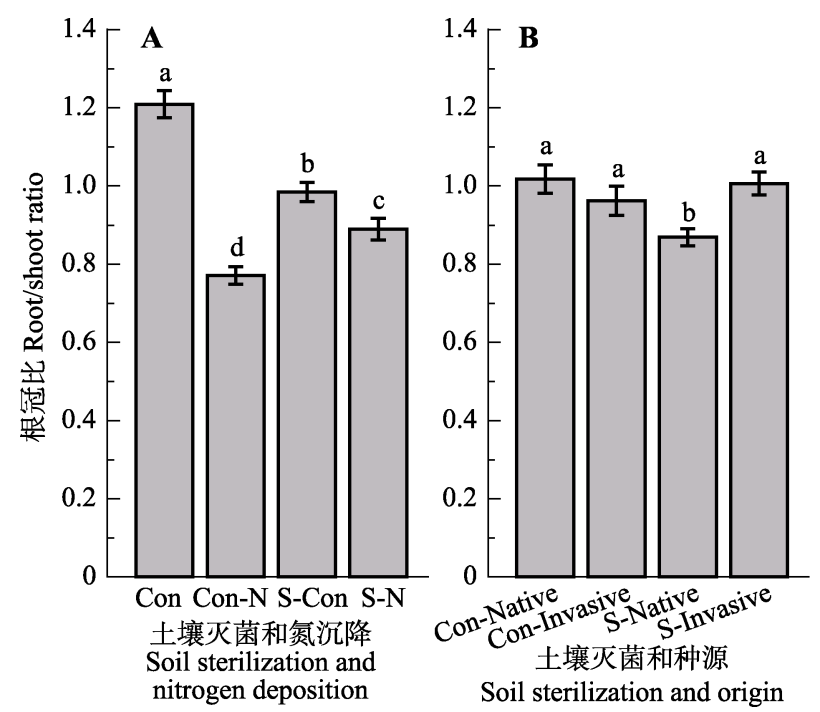

图4 不同处理对乌柏根冠比的影响(平均值土标准误差)。 Con, 对照; $N$, 氮沉降; $S$, 土壤灭菌; Native, 原产地; Invasive, 入侵地。相同小写字母表示没有显著差异 $(p>0.05$, LSD test)。

Fig. 4 Effect of different treatments on root/shoot ratio of Triadica sebifera (mean $\pm S E$ ). Con, control; N, nitrogen deposition; S, soil sterilization. Same lowercase letters indicate no significant differences ( $p>0.05, L S D$ test).
量(图3A)、茎生物量(图3C)和地上生物量(图3F), 降 低了根生物量(图3G)和RSR (图4A)。Yang等(2015) 的研究也表明施肥能显著降低乌柏的 $R S R$, 本试验 结果与之吻合, 说明氮沉降使乌柏对资源的分配发 生了改变, 更多的资源分配至地上部分, 特别是叶 片器官。

\subsection{UV-B辐射与土壤微生物之间没有显著的交互 作用}

土壤灭菌对株高(图1A)、叶片数(图2A)、叶面 积(图2C)、SLA (图2E)、叶生物量(图3A)、茎生物量 (图3C)、根生物量(图3E)、地上生物量(图3G)和 $R S R$ (图4A)都有显著负效应, 说明乌柏和土壤微生 物之间具有显著的正效应, 土壤灭菌处理消除了这 一正效应。但是, UV-B辐射与土壤灭菌之间没有显 著的交互作用(表2, 表3), 说明UV-B辐射在一定范 围内并没有影响土壤微生物对乌柏生长的影响。

\section{3 氮沉降没有减缓UV-B辐射对乌柏的胁迫作用}

UV-B辐射显著增加了乌柏叶生物量(图3B)和 叶片数(图2B), 降低了SLA (图2D)。叶片数增加可能 导致相互遮蔽以降低UV-B辐射对单体叶片的伤害 (李元等, 2015)。叶生物量增加, 表明资源分配发生 了改变, 可能是诱发了乌柏叶片抗UV-B辐射防御 (如叶片增厚、蜡质含量增加、黄酮类等次级代谢产 物增加, 栅栏组织细胞数目增加等)(王海霞和刘文 哲, 2011; Ballaré, 2014; 陈慧泽和韩榕, 2015; 李良 博等, 2015; 李元等, 2015; Suchar \& Robberecht, 2016)。此外, 高 $S L A$ 与低叶片成本投入和高光合 $\mathrm{N}$ 利用效率有关(Feng et al., 2008)。UV-B辐射显著降 低了SLA, 表明UV-B辐射增加了乌柏叶片成本投入 并降低了光合 $\mathrm{N}$ 利用效率。综合表明UV-B辐射胁迫 诱发了乌柏形态学防御。但是, UV-B辐射与氮沉降 之间并没有显著的交互作用(表2, 表3), 说明氮沉 降在一定范围内并没有减缓UV-B辐射对乌柏的胁迫 作用。

\section{4 入侵地乌相种群比原产地乌相种群更具优势}

入侵地乌柏相比于原产地乌柏具有较高的株 高(图1B)、茎生物量(图3D)和总生物量(图3H), 这与 Zou等(2007)的研究结果一致, 表明乌柏在入侵地 已经进化出了较强的生长性能。土壤灭菌对原产地 乌柏根生物量的负效应高于对入侵地乌柏的负效应 (-63.27\% vs. -53.64\%)(图3E), 说明原产地土壤微生 物对根系生长具有促进作用且原产地乌柏相比于入 
侵地乌柏更加依赖于原产地土壤有益微生物。此外, 虽然对照条件下原产地乌柏和入侵地乌柏 $R S R$ 没有 差异, 但是土壤灭菌条件下, 入侵地乌柏较之原产 地乌柏具有较高的 $R S R(+15.81 \%)$ 且土壤灭菌只对 原产地乌柏 $R S R$ 具有显著负效应, 对入侵地乌柏 $R S R$ 没有影响(图4B), 同样说明原产地乌柏相比于 入侵地乌柏更加依赖于原产地土壤有益微生物, 而 入侵地乌柏在一定程度上却摆脱了对原产地土壤有 益微生物的依赖。然而, 土壤灭菌对原产地乌柏 $L A R$ 的负效应却低于对入侵地乌柏的负效应 (-15.97\% vs. $-30.53 \%)$ (图2G), 说明原产地土壤微 生物对乌柏 $L A R$ 具有促进作用且入侵地乌柏相比于 原产地乌柏更加依赖于原产地土壤有益微生物。这 可能是入侵地乌柏和入侵地土壤的协同进化的结果， 弱化了入侵地乌柏在 $R S R$ 和根生物量方面对原产地 土壤微生物的依赖性, 但是增强了在 $L A R$ 方面的依 赖性。换言之, 入侵地乌柏在入侵地有选择性的增 强或弱化与某些有益微生物的协同互惠能力, 使之 具有较强的入侵潜力。Zhang等(2013)的研究表明土 壤灭菌处理会降低乌柏叶生物量和 SLA, 本试验结 果与之一致。然而, Nijjer等(2007)在美国乌柏入侵地 的试验结果却表明: 乌柏根际能够迅速累积有害微 生物群落, 与土壤微生物之间存在显著的负效应, 与本试验结果相反, 可能与本试验使用的是非根际 土, 病原菌引起的乌柏与土壤微生物间的负效应较 弱有关。本研究证实了UV-B辐射增强、氮沉降加剧 和土壤微生物在乌柏入侵过程中的潜在作用。

\section{基金项目 国家自然科学基金(41501317)。}

致谢 感谢江西农业大学科研启动基金(9232305172) 和博士后科研基金(9232306047)资助。感谢美国莱 斯大学Evan Siemann教授, 中国科学院武汉植物园 丁建清教授协助采集不同种群乌柏种子。

\section{参考文献}

Ballaré CL (2014). Light regulation of plant defense. Annual Review of Plant Biology, 65, 335-363.

Blossey B, Notzold R (1995). Evolution of increased competitive ability in invasive nonindigenous plants: A hypothesis. Journal of Ecology, 83, 887-889.

Bradley BA, Blumenthal DM, Wilcove DS, Ziska LH (2010). Predicting plant invasions in an era of global change. Trends in Ecology \& Evolution, 25, 310-318.

Callaway RM, Bedmar EJ, Reinhart KO, Silvan CG, Klironomos J (2011). Effects of soil biota from different ranges on Robinia invasion: Acquiring mutualists and escaping pathogens. Ecology, 92, 1027-1035.

Chen BM, Peng SL, Wu XP, Wang PL, Ma JX (2016). A bibliometric analysis of researches on topics related to the ecological damage caused by and risk assessments of exotic invasive species from 1995 to 2014. Acta Ecologica Sinica, 36, 1-9. (in Chinese with English abstract) [陈宝 明, 彭少麟, 吴秀平, 王鹏龙, 马建霞 (2016). 近20年 外来生物入侵危害与风险评估研究的文献计量分析. 生态学报, 36, 1-9.]

Chen HZ, Han R (2015). Plants respond to UV-B radiation: A review. Chinese Bulletin of Botany, 50, 790-801. (in Chinese with English abstract) [陈慧泽, 韩榕 (2015). 植物 响应UV-B辐射的研究进展. 植物学报, 50, 790-801.]

Chen T, Liu WL, Zhang CB, Wang J (2012). Effects of Solidago canadensis invasion on dynamics of native plant communities and their mechanisms. Chinese Journal of Plant Ecology, 36, 253-261. (in Chinese with English abstract) [陈殁, 刘文莉, 张崇邦, 王江 (2012). 加拿大一 枝黄花入侵对本土植物群落动态的影响及其机制. 植 物生态学报, 36, 253-261.]

Davis MA, Grime JP, Thompson K (2000). Fluctuating resources in plant communities: A general theory of invasibility. Journal of Ecology, 88, 528-534.

Feng YL, Fu GL, Zheng YL (2008). Specific leaf area relates to the differences in leaf construction cost, photosynthesis, nitrogen allocation, and use efficiencies between invasive and noninvasive alien congeners. Planta, 228, 383-390.

Henery ML, Bowman G, Mráz P, Treier UA, Gex-Fabry E, Schaffner U, Müller-Schärer H (2010). Evidence for a combination of pre-adapted traits and rapid adaptive change in the invasive plant Centaurea stoebe. Journal of Ecology, 98, 800-813.

Hock M, Beckmann M, Hofmann RR, Bruelheide H, Erfmeier A (2015). Effects of UV-B radiation on germination characteristics in invasive plants in New Zealand. NeoBiota, 26, 21-37.

Huang JX, Xu X, Wang M, Nie M, Qiu SY, Wang Q, Quan ZX, Xiao M, Li B (2016). Responses of soil nitrogen fixation to Spartina alterniflora invasion and nitrogen addition in a Chinese salt marsh. Scientific Reports, 6, 20384. doi: 10.1038/srep20384.

Huang W, Carrillo JL, Ding JQ, Siemann E (2012). Interactive effects of herbivory and competition intensity determine invasive plant performance. Oecologia, 170, 373-382.

Huang W, Wang Y, Ding JQ (2013). A review of adaptive evolution of defense strategies in an invasive plant species, Chinese tallow (Triadica sebifera). Chinese Journal of Plant Ecology, 37, 889-900. (in Chinese with English abstract) [黄伟, 王毅, 丁建清 (2013). 入侵植物乌柏防御 策略的适应性进化研究. 植物生态学报, 37, 889-900.]

Ju RT, Li H, Shi ZR, Li B (2012). Progress of biological invasions research in China over the last decade. Biodiversity Science, 20, 581-611. (in Chinese with English abstract) [鞠 瑞亭, 李慧, 石正人, 李博 (2012). 近十年中国生物入

www.plant-ecology.com 
侵研究进展. 生物多样性, 20, 581-611.]

Kanakidou M, Myriokefalitakis S, Daskalakis N, Fanourgakis G, Nenes A, Baker AR, Tsigaridis K, Mihalopoulos N (2016). Past, present, and future atmospheric nitrogen deposition. Journal of the Atmospheric Sciences, 73, 2039-2047.

Keane RM, Crawley MJ (2002). Exotic plant invasions and the enemy release hypothesis. Trends in Ecology \& Evolution, 17, 164-170.

Lei YB, Xiao HF, Feng YL (2010). Impacts of alien plant invasions on biodiversity and evolutionary responses of native species. Biodiversity Science, 18, 622-630. (in Chinese with English abstract) [类延宝, 肖海峰, 冯玉龙 (2010). 外来植物入侵对生物多样性的影响及本地生物的进化 响应. 生物多样性, 18, 622-630.]

Li LB, Tang TX, Hai MR, Chen JW, Zhou P (2015). Response and molecular mechanisms of plants to enhanced UV-B radiation. Chinese Agricultural Science Bulletin, 31(13), 159-163. (in Chinese with English abstract) [李良博, 唐 天向, 海梅荣, 陈军文, 周平 (2015). 植物对UV-B辐射 增强的响应及其分子机制. 中国农学通报, 31(13), 159163.]

Li LB, Zhang LG, Tang TX, Hai MR, Zhou P (2016). Influence of enhanced UV-B on physiological characteristics of Eupatorium adenophorum L. and Artemisia argyi Levl. Acta Botanica Boreali-Occidentalia Sinica, 36, 343-352. (in Chinese with English abstract) [李良博, 张连根, 唐天 向, 海梅荣, 周平 (2016). UV-B辐射增强对紫茎泽兰和 艾草抗性生理特性的影响. 西北植物学报, 36, 343-352.]

Li Y, Li X, He YM, Xia Y, Zu YQ (2015). Effect and evaluation of enhanced UV-B radiation on interactions between plant and pathogen. Plant Physiology Journal, 51, 15571566. (in Chinese with English abstract) [李元, 李想, 何 永美, 夏杨, 祖艳群 (2015). UV-B辐射增强对植物-病 原菌互作体系的影响及评价. 植物生理学报, 51, 1557-1566.]

Liu J, Li JM, Yu H, He WM, Yu FH, Sang WG, Liu GF, Dong M (2010). The relationship between functional traits and invasiveness of alien plants. Biodiversity Science, 18, 569576. (in Chinese with English abstract) [刘建, 李钧敏, 余 华, 何维明, 于飞海, 桑卫国, 刘国方, 董鸣 (2010). 植 物功能性状与外来植物入侵. 生物多样性, 18, 569-576.]

Lu RK (2000). Methods of Soil Agricultural Chemical Analysis. China Agriculture Scientech Press, Beijing. (in Chinese) [鲁如坤 (2000). 土壤农业化学分析方法. 中国农业科 技出版社, 北京.]

Ni Y, Song C, Li JN (2015). Effect of enhanced ultraviolet-B radiation on epicuticular wax in Arabidopsis thaliana. Acta Ecologica Sinica, 35, 1505-1512. (in Chinese with English abstract) [倪郁, 宋超, 李加纳 (2015). UV-B辐射增 强对拟南芥表皮蜡质的影响. 生态学报, 35, 1505-1512.]

Nijjer S, Rogers WE, Siemann E (2007). Negative plant-soil feedbacks may limit persistence of an invasive tree due to rapid accumulation of soil pathogens. Proceedings of the
Royal Society B: Biological Sciences, 274, 2621-2627.

Suchar VA, Robberecht R (2016). Integration and scaling of UV-B radiation effects on plants: From molecular interactions to whole plant responses. Ecology and Evolution, 1-19.

van der Putten WH, Klironomos JN, Wardle DA (2007). Microbial ecology of biological invasions. The ISME Journal, 1, 28-37.

Wan FH, Liu WX, Guo JY, Qiang S, Li BP, Wang JJ, Yang GQ, Niu HB, Gui FR, Huang WK, Jiang ZL, Wang WQ (2011). Invasive mechanism and control strategy of $\mathrm{Ag}$ eratina adenophora (Sprengel). Scientia Sinica Vitae, 41, 13-21. (in Chinese) [万方浩, 刘万学, 郭建英, 强胜, 李 保平, 王进军, 杨国庆, 牛红榜, 桂富荣, 黄文坤, 蒋智 林, 王文琪 (2011). 外来植物紫茎泽兰的入侵机理与控 制策略研究进展. 中国科学: 生命科学, 41, 13-21.]

Wang H, Ma XC, Zhang L, Zou JW, Siemann E (2016). UV-B has larger negative impacts on invasive populations of Triadica sebifera but ozone impacts do not vary. Journal of Plant Ecology, 9, 61-68.

Wang HX, Liu WZ (2011). Effects of enhanced UV-B radiation on leaf morphology and structure and pigment contents of Camptotheca acuminata. Chinese Agricultural Science Bulletin, 27(5), 209-213. (in Chinese with English abstract) [王海霞, 刘文哲 (2011). UV-B辐射增强对喜树 叶片色素含量和形态结构的影响. 中国农学通报, 27(5), 209-213.]

Williamson CE, Zepp RG, Lucas RM, Madronich S, Austin AT, Ballaré CL, Norval M, Sulzberger B, Bais AF, McKenzie RL, Robinson SA, Häder DP, Paul ND, Bornman JF (2014). Solar ultraviolet radiation in a changing climate. Nature Climate Change, 4, 434-441.

Wu H, Ding JQ (2014). Recent progress in invasion ecology. Chinese Science Bulletin, 59, 438-448. (in Chinese) [吴吴, 丁建清 (2014). 入侵生态学最新研究动态. 科学通报, 59, 438-448.]

Yang Q, Li B, Siemann E (2015). The effects of fertilization on plant-soil interactions and salinity tolerance of invasive Triadica sebifera. Plant and Soil, 394, 99-107.

Zhang L, Zhang Y, Wang H, Zou JW, Siemann E (2013). Chinese tallow trees (Triadica sebifera) from the invasive range outperform those from the native range with an active soil community or phosphorus fertilization. PLOS ONE, 8, e74233. doi: 10.1371/journal.pone.0074233.

Zou J, Roger WE, Siemann E (2007). Differences in morphological and physiological traits between native and invasive populations of Sapium sebiferum. Functional Ecology, 21, 721-730.

Zou JW, Roger WE, DeWalt SJ, Siemann E (2006). The effect of Chinese tallow tree (Sapium sebiferum) ecotype on soil-plant system carbon and nitrogen processes. Oecologia, 150, 272-281.

责任编委: 何维明 责任编辑: 李 敏 


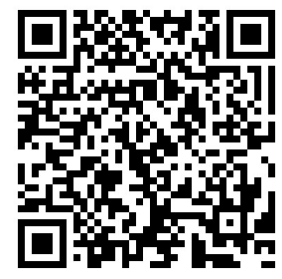

扫码向作者提问 